Published in final edited form as:

Environ Res. 2016 August ; 149: 15-22. doi:10.1016/j.envres.2016.04.031.

\title{
Does Total Antioxidant Capacity Modify Adverse Cardiac Responses Associated with Ambient Ultrafine, Accumulation Mode, and Fine Particles in Patients Undergoing Cardiac Rehabilitation?
}

\author{
Meng Wang ${ }^{1}$, Mark J. Utell ${ }^{2}$, Alexandra Schneider ${ }^{3}$, Wojciech Zareba ${ }^{4}$, Mark W. Frampton ${ }^{2}$, \\ David Oakes $^{5}$, Philip K. Hopke ${ }^{6}$, Jelani Wiltshire ${ }^{5}$, Cathleen Kane ${ }^{1}$, Annette Peters ${ }^{3}$, \\ Susanne Breitner ${ }^{3}$, David Chalupa ${ }^{2}$, and David Q. Rich ${ }^{1}$ \\ ${ }^{1}$ Division of Epidemiology, Department of Public Health Sciences, University of Rochester Medical \\ Center, Rochester, New York, USA \\ 2Division of Pulmonary and Critical Care, Department of Medicine, University of Rochester \\ Medical Center, Rochester, New York, USA \\ ${ }^{3}$ Institute of Epidemiology II, Helmholtz Zentrum Muenchen - German Research Center for \\ Environmental Health, Neuherberg, Germany \\ ${ }^{4}$ Division of Cardiology, Department of Medicine, University of Rochester Medical Center, New \\ York, USA \\ ${ }^{5}$ Department of Biostatistics and Computational Biology, University of Rochester Medical Center, \\ Rochester, New York, USA \\ ${ }^{6}$ Center for Air Resources Engineering and Science, Clarkson University, Potsdam, NY, USA
}

\section{Abstract}

Background-Previous studies suggest that pathways reducing oxidative stress may have a protective effect against adverse cardiac responses associated with ambient PM. However, few studies have directly assessed total antioxidant capacity (TAC) as a potential effect modifier of cardiac responses to increased ambient PM.

Objectives-We examined if TAC modifies the association between ambient PM and markers of heart rate variability (HRV), repolarization, systemic inflammation, and systolic blood pressure (SBP) in post-infarction patients.

Methods-We recruited 76 patients with a recent coronary event (myocardial infarction or unstable angina) who participated in a cardiac rehabilitation program from June 2006 to November 2009 in Rochester, New York. Ambient fine particle $\left(\mathrm{PM}_{2.5}, \underline{\Omega} .5 \mu \mathrm{m}\right.$ in aerodynamic

*Correspondence to David Q. Rich, University of Rochester Medical Center, Department of Public Health Sciences, 265 Crittenden Blvd., CU 420644, Rochester, NY 14642 USA. Telephone: (585) 276-4119. Fax: (585) 424-1469. david_rich@urmc.rochester.edu. Publisher's Disclaimer: This is a PDF file of an unedited manuscript that has been accepted for publication. As a service to our customers we are providing this early version of the manuscript. The manuscript will undergo copyediting, typesetting, and review of the resulting proof before it is published in its final citable form. Please note that during the production process errors may be discovered which could affect the content, and all legal disclaimers that apply to the journal pertain. 
diameter), accumulation mode particle (AMP, 100-500nm) and ultrafine particle (UFP, 10-100nm) concentrations were measured continuously by fixed-site monitors. Markers of HRV and repolarization were measured by continuous Holter electrocardiogram (ECG) recordings before and during exercise sessions of the rehabilitation program. Blood pressure was measured and venous blood samples were collected before exercise to measure TAC and inflammation markers. We applied linear mixed models to assess changes in markers of HRV, repolarization, systemic inflammation, and SBP associated with increased PM concentrations in the low, medium and high TAC tertile groups, after adjusting for covariates including temperature, calendar time since the beginning of the study, visit number, month of year, and hour of day.

Results-Based on subject-visits with available TAC, we observed increases in SBP, C-reactive protein, and fibrinogen, and decreases in rMSSD (square root of the mean of the sum of the squared differences between adjacent normal to normal intervals)and SDNN (standard deviation of normal to normal beat intervals) associated with increased $\mathrm{PM}_{2.5}$, AMP and UFP in the previous 6 to 120 hour (e.g. change in SBP associated with each interquartile range (IQR) increase in $\mathrm{PM}_{2.5}$ lagged 0-5 hours was $1.27 \mathrm{mmHg}$ [95\%CI: 0.09-2.46mmHg)). However, we did not observe a consistent pattern of effect measure modification by TAC for any combination of pollutant and outcome (e.g. changes in SBP associated with each IQR increase in $\mathrm{PM}_{2.5}$ lagged 0-5 hours for the low, medium and high TAC tertile groups were $1.93 \mathrm{mmHg}$ [95\%CI: $0.23,3.63],-0.31 \mathrm{mmHg}$ [95\%CI: $-2.62,2.01$ ], and $1.29 \mathrm{mmHg}$ [95\%CI: $-0.64,3.21$ ], respectively. P for interaction=0.28).

Conclusions - In a post-infarction population, total antioxidant capacity does not appear to modify the association between biomarkers of heart rate variability, repolarization, systemic inflammation, and systolic blood pressure and ambient PM concentrations in the previous 6 to 120 hours.

\section{Keywords}

Particulate matter; total antioxidant capacity; heart rate variability; repolarization; inflammation

\section{INTRODUCTION}

Increases in ambient particulate matter air pollution (PM) over several hours and/or days have been associated with the triggering of myocardial infarction (MI) [1-3]. Several mechanisms have been proposed to mediate this response to air pollution including adverse changes in systolic blood pressure, systemic inflammation, heart rate variability and repolarization. Some studies have examined whether short term increases in PM are associated with adverse changes in biomarkers of these pathways, with some studies reporting adverse changes in biomarkers of these pathways (e.g. C-reactive protein [CRP], fibrinogen, standard deviation of normal to normal beat intervals [SDNN], square root of the mean of the sum of the squared differences between adjacent normal to normal intervals [rMSSD], and T-wave complexity) associated with increased PM in the previous few hours and days $[4,5]$.

Oxidative stress has been proposed as another mechanism by which PM may adversely affect cardiovascular health [4]. PM may shift the balance of reactive oxygen species/ antioxidant production towards an oxidative status, which may contribute to the development 
of cardiovascular disease [6]. Total antioxidant capacity (TAC) in blood is a relatively comprehensive assessment of antioxidant effects of different antioxidant molecules present in the blood, thus providing an integrated parameter of antioxidant capacity [7]. High levels of TAC could therefore be indicative of decreased susceptibility to oxidative damage [8]. Although some studies suggest that pathways reducing oxidative stress (e.g. certain genotype and dietary antioxidants) may have a protective effect against adverse changes in heart rate variability and other cardiovascular endpoints associated with ambient PM [9-14], no study to date has directly assessed TAC as a potential effect modifier of cardiovascular responses to increased ambient PM.

In our previous study of patients in a cardiac rehabilitation program in Rochester, New York, we reported that short term increases (5-120 hour) in ambient fine particle $\left(\mathrm{PM}_{2.5}, \mathcal{2} .5 \mu \mathrm{m}\right.$ in aerodynamic diameter), accumulation mode particle (AMP, 100-500nm) and ultrafine particle (UFP, 10-100nm) concentrations were associated with adverse changes in markers of heart rate variability (specifically, SDNN and rMSSD), repolarization (specifically, time from peak to end of T-wave), systemic inflammation (specifically, C-reactive protein and fibrinogen), and systolic blood pressure [15]. Using this same study population, we examined if TAC modified these associations. We hypothesized that increases in CRP, fibrinogen, SBP, and T-wave complexity, and decreases in SDNN and rMSSD associated with increased ambient PM concentrations would be muted or lessened for those with higher TAC levels compared to those with lower TAC levels.

\section{METHODS}

\subsection{Study population}

This population has been described previously [15]. Briefly, we recruited 76 patients with a recent coronary event (MI or unstable angina) in the University of Rochester Cardiac Rehabilitation Center (CR Center) from June 2006 to November 2009. These patients were referred by their cardiologists to participate in a cardiac rehabilitation program which included two rehabilitation sessions per week over a 10 week period ( 20 sessions per subject at most). At each session, patients were in the CR Center for 30-60 minutes before exercising. During this pre-exercise period, we collected a blood sample and placed a Holter monitor on each subject. The exercise session then started, after a 2-5 minute warm-up period which included gentle stretching. During the exercise session, patients exercised for 30-45 minutes using a bicycle, treadmill, or rowing machine chosen by each patient themselves as part of their rehabilitation program. After completion, patients had a "cool down" recovery period and then rested for 10 minutes. The same modality was used for each patient throughout the 10-week period.

Different from the previous analysis that used all subject-visits (up to 20 visits per subject) [15], we only included subject-visits with available TAC data (up to 10 visits per subject) for this analysis. We then excluded patients who were current smokers or nonsmokers living with an active smoker, those who had non-ischemic cardiomyopathy, type 1 diabetes, chronic atrial fibrillation, anemia, or left bundle branch block, those who had prosthetic heart valve or pacemaker, coronary bypass grafting within the last three months, or regular use of amiodarone. All patients lived within $19 \mathrm{~km}$ of the particle monitoring site at the CR 
center (median: $9 \mathrm{~km}$ ) and within $21 \mathrm{~km}$ of the New York State Department of Environmental Conservation (NYS DEC) particle monitoring site (median: $9 \mathrm{~km}$ ). This study was approved by the Research Subjects Review Board of the University of Rochester.

\subsection{Air pollution and weather assessments}

Air pollution and weather measurements have been described previously [15]. In short, concentrations of ultrafine particles (UFP; 10-100nm diameter) and accumulation mode particles (AMP; 100-500nm diameter) were measured at the CR monitoring site from June 2006 to November 2009 using a wide range particle spectrometer (model 1000XP; MSP Corporation, Shoreview, MN). This monitoring site is about $1500 \mathrm{~m}$ away from an interstate high-way beltway. A size range of 100-500nm was defined for AMP rather than the standard $100-1000 \mathrm{~nm}$ definition. Given that the majority of particles in the standard size range are smaller in mass and closer to the 100nm lower limit, the approximate definition used in this study should result in minimal difference in AMP concentration. Concentrations of $\mathrm{PM}_{2.5}$ were measured at the NYS DEC monitoring site in Rochester (approximately $1.2 \mathrm{~km}$ from the CR center) using a tapered element oscillating microbalance (ThermoFisher, Franklin, MA). Weather measurements including hourly temperature, relative humidity and barometric pressure were measured at the NYS DEC site.

\subsection{ECG outcome assessments}

Heart rate variability parameters and T-wave complexity (measure of T-wave morphology and repolarization) were measured by 3-lead (modified V2, V5, and AVF) Holter ECG recordings (Burdick Altair-Disc Holter recorder; Cardiac Science, Bothell, WA) across the pre-exercise (baseline), exercise and post-exercise periods at each visit (twice a week)[15]. Pre-exercise measurements were performed after a resting period during which patients seated for at least five minutes. In the present study, heart rate variability parameters including SDNN and rMSSD measured across the whole session (1-3 hours) were used in the analysis. Whole session recording provided more information on the overall behavior of heart rate and autonomic responses to daily conditions including the exercise. For $\mathrm{T}$ wave complexity, pre-exercise measurements were used to avoid noise caused by exercise. Principal component analysis was applied to quantify T-wave complexity, which was defined as the ratio of the second to first eigenvalues of the spatial T-wave vector (PCA ratio). The PCA ratio provides an estimate of the relative "fatness" of the three-dimensional T-wave loop relative to its peak amplitude and a higher PCA ratio (a fatter loop) reflects more complex repolarization [16].

Holter recordings were annotated first automatically by the commercial Holter scanning algorithm (Vision Premier Burdick Holter System; Cardiac Science) and then analyzed by a trained technician using custom-made programs at the University of Rochester Medical Center, which have been described previously [17, 18]. Based in part on Bigger et al.[19], filtering criteria eliminated two RR intervals after premature ventricular or atrial beats. To evaluate extreme values, we applied a post-processing approach. We examined the distribution of each ECG parameter for each period. Values were considered to be outliers if they were greater than the $99^{\text {th }}$ percentile or smaller than the first percentile in the study 
population. We then evaluated these outliers and determined whether the values were valid or not based on intra-lab ranges developed by a previous study [20].

\subsection{Blood pressure and blood sample measurements}

Blood pressure was measured at each visit (twice a week) by auscultation following the 5minute pre-exercise resting period with the arm supported at heart level. Based on the research protocol [15], three measurements were performed at each time and the average was used in the analyses. Venous blood samples were collected once weekly before exercise and were analyzed for fibrinogen and CRP in the Strong Memorial Hospital Clinical Laboratories (University of Rochester Medical Center, Rochester, NY).

\subsection{TAC measurements}

We applied a commercial assay, Cell Biolabs OxiSelect ${ }^{\mathrm{TM}}$ Total Antioxidant Capacity (TAC) Assay Kit (San Diego, USA), to measure TAC concentration in each blood sample in our laboratory at Rochester. The assay is based upon the reduction of copper (II) to copper (I) by antioxidants in the sample (e.g. uric acid). The copper (I) ion can further react with a coupling chromogenic reagent that produces a color with a maximum absorbance at $490 \mathrm{~nm}$. Samples were compared to a known concentration of uric acid standard, with results expressed as mM Uric Acid Equivalents. The copper assay method detects all classes of antioxidants including thiols, with marginal radical interference.

\subsection{Statistical analysis}

We first assessed the change in each outcome (systolic blood pressure, CRP, fibrinogen, rMSSD, SDNN, and T-wave complexity,) associated with each interquartile range (IQR) increase in pollutant concentrations ( $\mathrm{PM}_{2.5}$, AMP and UFP) in the previous 24 hours (lag hours 0-23), using subject-visits with available TAC data (up to 10 visits per subject). To account for dependencies between the repeated measurements within each subject, we applied mixed models with subjects entered as a random effect. All models were adjusted for the mean temperature in the previous 24 hours, calendar time since the beginning of the study, as well as indicator variables for visit number, month of year, and hour of day. Among all weather measurements, only temperature was included into the model because it was the only variable that consistently was a significant $(\mathrm{p}<0.05)$ predictor of the outcomes (strongest for lag hour 0-23). We then separately reran this model examining increases in each air pollutant in the lag hours 0-5, 24-47, 48-71, 72-95, and 96-119.

TAC tertiles ( 0.58 and $0.68 \mathrm{mM}$ Uric Acid Equivalents) were obtained based on a total of 656 blood measurements. Potential effect measure modification of the $\mathrm{PM}_{2.5} /$ systolic blood pressure association by TAC was assessed by adding indicator variables for tertiles of TAC (low, medium, high), and interaction terms of TAC tertile and $\mathrm{PM}_{2.5}$ to the models described above. From this model, we present the change in systolic blood pressure associated with each IQR increase in $\mathrm{PM}_{2.5}$ concentration in the previous 24 hours (lag hours 0-23), separately within those with low TAC levels, those with medium TAC levels, and those with high TAC levels. We then repeated this analysis for each combination of outcome (systolic blood pressure, CRP, fibrinogen, rMSSD, SDNN, and T-wave complexity) and PM air pollution ( $\mathrm{PM}_{2.5}$, UFP, and AMP). As a sensitivity analysis, we also examined effect 
modification by the average TAC value for each subject (i.e. one TAC value per subject rather than one TAC value per subject-visit). We log-transformed AMP and UFP in all analyses to reduce skewness, and log-transformed CRP and T-wave complexity based on residual plots to better fit model assumptions (linear relationship, equal variance, et al). All analyses were performed using SAS software (version 9.2; SAS institute Inc., Cary, NC) with $\mathrm{p}<0.05$ used to define statistical significance.

\section{RESULTS}

The study population has been described previously [15]. Briefly, 63 patients (83\%) completed all 20 visits, and 6 patients (8\%) completed less than 10 visits. The majority of patients were white (88\%) and male (67\%), with a mean age of 60 years (range: $36-80$ years). Eighty-seven percent of patients had a body mass index greater than $25 \mathrm{~kg} / \mathrm{m}^{2}$ and $47 \%$ patients were former smokers. Most patients had a history of myocardial infarction (59\%), hypertension (59\%), or a prior stent $(86 \%)$. Use of beta blocker, angiotensinconverting-enzyme inhibitors, and statins was common in this population. Distributions of each outcome measured across the study period (only subject-visits with available TAC data were included) are provided in supplementary Table 1.

Distributions of hourly (at lag hour 0) and 24-hour mean (at lag hours 0-23) concentrations of each pollutant and temperature before each clinical visit are shown in Table 1. Pollutant concentrations were relatively low compared to a number of other U.S. and international locations[21]. For 24-hour mean concentrations, AMP was moderately correlated with $\mathrm{PM}_{2.5}(\mathrm{r}=0.63)$ and UFP ( $\left.\mathrm{r}=0.60\right)$, but $\mathrm{PM}_{2.5}$ and UFP were poorly correlated ( $\left.\mathrm{r}=0.16\right)$. Temperature was poorly correlated with UFP ( $\mathrm{r}=-0.13)$, and was not correlated with $\mathrm{PM}_{2.5}$ and $\operatorname{AMP}(\mathrm{r}=0.05$ and 0.02 , respectively).

Table 2 shows changes in each outcome associated with each IQR increase in $\mathrm{PM}_{2.5}, \log$ AMP, and log UFP concentrations measured in the previous 120 hours before the clinical visit, for this smaller subset of subject-visits with TAC data. For most outcomes, our findings showed similar patterns of response to increased pollutant concentrations as those found previously [15]. We observed increased systolic blood pressure associated with each IQR increase in $\mathrm{PM}_{2.5}, \log \mathrm{AMP}$, and $\log$ UFP for most lag periods. The largest increase was observed at lag hours 0-5 for $\mathrm{PM}_{2.5}(1.27 \mathrm{mmHg}$, 95\% CI: 0.09 to $2.46 \mathrm{mmHg})$, at lag hours 0-23 for log AMP (1.48 mmHg, 95\% CI: 0.09 to $2.86 \mathrm{mmHg}$ ), and at lag hours $24-47$ for log UFP (1.60 mmHg, 95\%CI: 0.32 to $2.89 \mathrm{mmHg}$ ). Increased pollutant concentrations were also associated with elevated log CRP and fibrinogen at most lag periods, although only a few associations were statistically significant. Each IQR increase in $\mathrm{PM}_{2.5}$ in the previous 24 hours (lag hours 0-23) was significantly associated with a 0.065 (95\%CI: 0.003 to 0.126 ) increase in $\log \mathrm{CRP}$ (which was a $6.69 \%$ increase in CRP, 95\%CI: $0.34 \%-13.44 \%$ ), whereas IQR increases in log AMP and log UFP lagged 24-47 hours were significantly associated with $0.10 \mathrm{~g} / \mathrm{L}$ (95\%CI: 0.02 to $0.18 \mathrm{~g} / \mathrm{L}$ ) and $0.07 \mathrm{~g} / \mathrm{L}$ (95\%CI: 0.00 to $0.14 \mathrm{~g} / \mathrm{L}$ ) increases in fibrinogen, respectively.

We observed decreased rMSSD associated with each IQR increase in log AMP and log UFP for all lag periods (Table 2). IQR increases in log AMP lagged 72-95 hours and in log UFP 
lagged 0-5, 0-23, 24-47, 48-71, and 72-95 hours were all significantly associated with decreased rMSSD. The largest decrease was observed for log UFP lagged 72-95 hours (-7.48ms, 95\%CI: -10.77 to $-4.20 \mathrm{~ms}$ ). Although not significant, we observed decreases in SDNN (range: $-2.14 \mathrm{~ms}$ to $-0.14 \mathrm{~ms}$ ) associated with each IQR increase in all three pollutants within the previous 48 hours (lag 0-5 hour, lag 0-23 hour, and lag 25-47 hour). For log Twave complexity, there was no significant change associated with any pollutants, although we did observe non-statistically significant decreases (range: -0.059 to -0.010) in log T-wave complexity (which means a $0.97 \%-5.72 \%$ decrease in T-wave complexity) within the previous 24 hours for each of the three pollutants.

The distribution of TAC in the low, medium and high tertile groups are presented in Table 3. Intra-class correlation coefficient for TAC was 0.58 , which indicates moderate withinsubject correlation. For each pollutant/outcome combination, we identified from table 2 the largest effect estimate (e.g. the largest change in SBP associated with increased $\mathrm{PM}_{2.5}$ concentration was for lag hours 0-5). In Figures 1-6 we presented the estimated changes in these outcomes associated with each IQR increase in these lagged pollutant concentrations, separately for the low, medium, and high TAC tertile group. We did not observe a consistent pattern of effect measure modification by TAC (i.e. smaller changes in an outcome associated with increased air pollutant concentrations in the higher TAC groups compared to the lower TAC groups) for any combination of pollutant ( $\mathrm{PM}_{2.5}$, UFP, or AMP) and outcome (systolic blood pressure, CRP, fibrinogen, SDNN, rMSSD, or T-wave complexity). We also examined other lag periods for each combination of pollutant and outcome, and examined whether the average TAC value for each subject across all visits modified any pollutant/ outcome association. However, the results were consistent with those presented in the main analysis, in that no pattern of effect modification was observed (data not shown).

\section{DISCUSSION}

To our knowledge, this is the first study assessing TAC as a potential effect modifier of the association between short term (within a few hours and days) increases in ambient $\mathrm{PM}_{2.5}$, AMP, and UFP concentrations, and biomarkers of heart rate variability, repolarization, systemic inflammation, and systolic blood pressure. As in our previous analysis, we found increased $\mathrm{PM}_{2.5}$, UFP, and AMP concentrations in the previous 6 to 120 hours were associated with adverse changes in these biomarkers in a population of post-infarction patients. However, we did not find effect measure modification by TAC of any of these associations. Our findings suggest that blood TAC quantified by an assay based upon the reduction of copper (II) to copper (I) by antioxidants does not protect against the adverse cardiovascular responses induced by short term increases in ambient PM pollution concentrations in post-infarction patients participating in a cardiac rehabilitation program.

Reduced heart rate variability, which reflects cardiac autonomic imbalance, has been independently associated with worse cardiovascular prognosis [22, 23]. Previous studies have shown effect modification of the relationship between PM and heart rate variability by dietary antioxidants and genes that modulate endogenous oxidative stress [9-13]. In 549 elderly men from the Normative Aging Study, Baccarelli et al. observed decreased SDNN in subjects with lower intakes of B6 (-13.1\%, 95\%CI: $-20.0 \%$ to $-5.5 \%), \mathrm{B} 12(-12.2 \%, 95 \% \mathrm{CI}$ : 
$-19.1 \%$ to $-4.7 \%)$, or methionine (-11.9\%, $95 \% \mathrm{CI}:-18.9 \%$ to $-4.1 \%)$ associated with each $10 \mu \mathrm{g} / \mathrm{m}^{3}$ increase in $\mathrm{PM}_{2.5}$ concentration in the 48 hours before the measurement. However, no such association with increased $\mathrm{PM}_{2.5}$ concentration was observed in the higher B6, B12, or methionine intake groups [9]. Based on the same population, a study by Chahine et al. found that functional genetic variations in glutathione S-transferase M1 (GSTM1) and heme oxygenase-1 (HMOX-1, both of which are related to defenses against oxidative stress) modified the association between $\mathrm{HRV}$ and $\mathrm{PM}_{2.5}$ concentrations measured in the previous 48 hours [11]. In their study, no significant change in SDNN, high frequency (HF), or low frequency (LF) HRV associated with increased $\mathrm{PM}_{2.5}$ in the 48 hours before measurement was observed in subjects with GSTM1 or the short repeat variant of HMOX-1. However, significant decreases in these HRV biomarkers were observed in subjects with a GSTM1 deletion or any long repeat of HMOX-1. In subjects with the GSTM1 deletion and the HMOX-1 long repeat, SDNN decreased by $13 \%$ (95\%CI: $-21 \%$ to $-4 \%)$, HF decreased by $28 \%$ (95\%CI: $-43 \%$ to $-9 \%$ ), and LF decreased by $20 \%$ (95\%CI: $-35 \%$ to $-3 \%$ ) per $10 \mu \mathrm{g} / \mathrm{m}^{3}$ increase in $\mathrm{PM}_{2.5}$ concentration, whereas in subjects with GSTM1 and HMOX-1 short repeat, changes in SDNN, HF and LF per $10 \mu \mathrm{g} / \mathrm{m}^{3}$ were $29 \%$ (95\%CI: $-12 \%$ to $88.2 \%$ ), $46 \%$ (95\%CI: $-44 \%$ to $275 \%$ ), and $68.5 \%$ (-23\% to $270 \%$ ), respectively.

Different from the above studies that focused on genes or dietary supplements related to antioxidant defense, we used blood TAC as a direct measure of antioxidant capacity. We did not observe effect modification by TAC on the associations between ambient PM including $\mathrm{PM}_{2.5}$, AMP and UFP, and two time-domain measures of HRV (SDNN and rMSSD) in a post-infarction population. It should be noted that there has been controversy regarding measuring TAC in plasma to quantify in vivo antioxidant capacity [8, 24-27]. Given that blood samples were measured by means of in vitro test systems, the TAC assay cannot account for in vivo antioxidant enzyme activities in plasma, which play an important role in human antioxidant defense system $[8,25]$. Furthermore, antioxidants can exert effects by different mechanisms[27]. The TAC assay used in the present study was based on a radical scavenging mechanism. However, there is no single method which correctly produces a comprehensive evaluation of total antioxidant capacity. The results often depend on the method employed and the arbitrarily selected oxidant generator [24-27]. Hence, our negative result might be explained by failure to adequately measure the individuals' true total antioxidant capacity. Additionally, differences in SDNN measurement may also contribute to the inconsistent findings. In the Normative Aging Study, SDNN was measured for 7 minutes while the subject was seated. In the present study we used whole session ECG recordings that include both resting periods and exercise periods. Including exercise period in the recording, likely provided HRV parameters that more closely resemble real-world conditions in which sympathetic stimuli exist, whereas resting recordings only provide HRV parameters unaffected by sympathetic stimuli. Finally, it is also possible that TAC values in this post-MI population were lower and less variable than those in the general population, which makes it difficult to detect potential effect modifications by TAC and thus may contribute to the null finding. Similar analyses may need to be done in populations of subjects without a recent MI.

For other endpoints (repolarization, inflammation, and blood pressure), few human studies have investigated potential effect modification of the association between these outcomes 
and ambient air pollutants by antioxidant defense. The Veterans Affairs Normative Aging Study reported larger increases in QT intervals (an electrocardiographic marker of the duration of repolarization) associated with increased traffic-related pollutant concentrations during the 10 hour before visit in participants with higher scores of genetic susceptibility to oxidative stress compared to participants with lower scores[28]. In participants with higher scores, changes in QT intervals associated with each IQR increase in black carbon, nitrogen dioxide, and carbon monoxide concentrations were $3.85 \mathrm{~ms}$ (95\%CI: 0.78 to $6.93 \mathrm{~ms}$ ), $3.50 \mathrm{~ms}$ (95\%CI: -1.86 to $8.87 \mathrm{~ms}$ ), and $6.52 \mathrm{~ms}$ (95\%CI: 0.49 to $12.55 \mathrm{~ms}$ ), respectively; whereas In participants with lower scores, changes in QT intervals associated with each IQR increase in black carbon, nitrogen dioxide, and carbon monoxide concentrations were $-0.56 \mathrm{~ms}$ (95\%CI: -3.90 to $2.78 \mathrm{~ms}$ ), $0.29 \mathrm{~ms}$ (95\% CI: -4.56 to $5.14 \mathrm{~ms}$ ), and $3.94 \mathrm{~ms}$ (95\%CI: -1.28 to $9.16 \mathrm{~ms}$ ), respectively. This study found no association between QT interval and $\mathrm{PM}_{2.5}$ exposure. It has been recognized that duration is only one aspect of repolarization. Morphological aberrations also carry major clinical implications for abnormal repolarization[29]. Therefore, in the present study we focused on $\mathrm{T}$ wave complexity, which is a measure of $\mathrm{T}$ wave morphology. We did not observe significant associations between $\mathrm{T}$ wave complexity and any of these three pollutants. Neither did we observe effect measure modification of these associations by TAC. In another research by Mordukhovich et al based on the Normative Aging Study, no evidence of effect modification of the association between blood pressure and black carbon by gene variants related to antioxidative defense was found [30]. This study did not observe any association between blood pressure and $\mathrm{PM}_{2.5}$ exposure. Although we observed increased systolic blood pressure associated with IQR increases in all three pollutants, we did not observe any effect modification by TAC of these associations. In addition to the above endpoints assessed in the present study, a panel study by Schneider et al reported greater decrements in flow-mediated dilatation, a measure of endothelial function, induced by $\mathrm{PM}_{2.5}$ exposure at lag day 0 among participants with the null polymorphism of GSTM1, suggesting an effect measure modification by oxidative potential of the association between $\mathrm{PM}_{2.5}$ exposure and endothelial function[31].

Our study has several limitations that should be considered when making inference. First, we used data from monitoring stations to assign exposure to all subjects living within this area regardless of the distance from the monitoring station to the subject's residence and each subject's daily mobility. However, this measurement error is likely to be a combination of classical and Berkson error. While classical error would bias the effect estimates towards the null, Berkson error would only decrease the precision of effect estimates without introducing bias. Therefore, the combined influence of these two types of error is likely to be an underestimate of the main effect of air pollution on cardiovascular responses[32]. This measurement error and resulting bias may be even greater for UFP, since it is generally more spatially variable than $\mathrm{PM}_{2.5}$ and AMP. Second, our sample size may have resulted in insufficient statistical power to detect an interaction effect. However, the negative findings were unlikely to be explained completely by a lack of power since the effect estimates showed no consistent pattern or trend of smaller adverse outcome changes associated with increased $\mathrm{PM}_{2.5}$, UFP, or AMP in the high TAC tertile groups compared to the low or middle TAC groups. Additionally, type I error in the assessment of main effect might be inflated due to multiple comparisons. Third, as discussed above, blood TAC may not accurately reflect in 
vivo antioxidant capacity. However, given that there is no ideal method to quantify total antioxidant capacity, blood TAC is still considered as a reasonable biomarker to provide information regarding individuals' overall antioxidant capacity[7]. Finally, our study population only included post-infarction patients participating in a Cardiac Rehabilitation program. Thus our subjects may not be representative of the general population.

\section{CONCLUSION}

In this panel study of post-infarction patients who participated in a cardiac rehabilitation program, we did not find effect measure modification by TAC on any associations between ambient $\mathrm{PM}_{2.5}$, AMP and UFP concentrations, in the previous 6 to 120 hours, and biomarkers of heart rate variability, repolarization, systemic inflammation, and systolic blood pressure. More studies with improved methods to quantify total antioxidant capacity are needed to confirm or refute our findings.

\section{Supplementary Material}

Refer to Web version on PubMed Central for supplementary material.

\section{Acknowledgments}

This work was supported by the New York State Energy Research and Development Authority (contract 8650), the U.S. Environmental Protection Agency (EPA) through a grant from the Science to Achieve Results (RD83241501), a Syracuse Center of Excellence CARTI (Collaborative Activities for Research and Technology Innovation) project award, which was supported by a grant from the U.S. EPA (X-83232501-0), the Electric Power Research Institute (EPRI agreement W06325), the Health Effects Institute (HEI agreement \# 4908 RFPA 10-3/12-2), and the National Institute of Environmental Health Sciences Center (P30 ES01247). EPRI is primarily supported by the electric industry in the United States and abroad. EPRI is an independent nonprofit 501(c)(3) organization that funds external research at a number of universities and institutes worldwide. Although the research described in this article has been funded in part by the U.S. EPA, it has not been subjected to the Agency's required peer and policy review. Therefore, it does not necessarily reflect the views of the U.S. EPA and no official endorsement should be inferred. Jelani Wiltshire's work was supported by training grant T32-ES007271.The above funding sources had no involvement in study design, data collection, analysis and interpretation of data, as well as in the writing of the report and decision to submit the article for publication. We thank all study subjects for their enthusiastic participation.

Finding sources

This work was supported by the New York State Energy Research and Development Authority (contract 8650), the U.S. Environmental Protection Agency (EPA) through a grant from the Science to Achieve Results (RD83241501), a Syracuse Center of Excellence CARTI (Collaborative Activities for Research and Technology Innovation) project award, which was supported by a grant from the U.S. EPA (X-83232501-0), the Electric Power Research Institute (EPRI agreement W06325), the Health Effects Institute (HEI agreement \# 4908 RFPA 10-3/12-2), and the National Institute of Environmental Health Sciences Center (P30 ES01247). EPRI is primarily supported by the electric industry in the United States and abroad. EPRI is an independent nonprofit 501(c)(3) organization that funds external research at a number of universities and institutes worldwide. Jelani Wiltshire's work was supported by training grant T32-ES007271

University of Rochester Research Subjects Review Board approval number: RSRB00013732

\section{References}

1. Mustafic H, et al. Main air pollutants and myocardial infarction: a systematic review and metaanalysis. Jama. 2012; 307(7):713-21. [PubMed: 22337682] 
2. Gardner B, et al. Ambient fine particulate air pollution triggers ST-elevation myocardial infarction, but not non-ST elevation myocardial infarction: a case-crossover study. Part Fibre Toxicol. 2014; 11:1. [PubMed: 24382024]

3. Bhaskaran K, et al. Effects of air pollution on the incidence of myocardial infarction. Heart. 2009; 95(21):1746-59. [PubMed: 19635723]

4. Brook RD, et al. Particulate matter air pollution and cardiovascular disease: An update to the scientific statement from the American Heart Association. Circulation. 2010; 121(21):2331-78. [PubMed: 20458016]

5. Ruckerl R, et al. Health effects of particulate air pollution: A review of epidemiological evidence. Inhal Toxicol. 2011; 23(10):555-92. [PubMed: 21864219]

6. Dalle-Donne I, et al. Biomarkers of oxidative damage in human disease. Clin Chem. 2006; 52(4): 601-23. [PubMed: 16484333]

7. Ghiselli A, et al. Total antioxidant capacity as a tool to assess redox status: critical view and experimental data. Free Radic Biol Med. 2000; 29(11):1106-14. [PubMed: 11121717]

8. Young IS. Measurement of total antioxidant capacity. J Clin Pathol. 2001; 54(5):339. [PubMed: 11328830]

9. Baccarelli A, et al. Cardiac autonomic dysfunction: effects from particulate air pollution and protection by dietary methyl nutrients and metabolic polymorphisms. Circulation. 2008; 117(14): 1802-9. [PubMed: 18378616]

10. Romieu I, et al. The effect of supplementation with omega-3 polyunsaturated fatty acids on markers of oxidative stress in elderly exposed to PM(2.5). Environ Health Perspect. 2008; 116(9): 1237-42. [PubMed: 18795169]

11. Chahine T, et al. Particulate air pollution, oxidative stress genes, and heart rate variability in an elderly cohort. Environ Health Perspect. 2007; 115(11):1617-22. [PubMed: 18007994]

12. Schwartz J, et al. Glutathione-S-transferase M1, obesity, statins, and autonomic effects of particles: gene-by-drug-by-environment interaction. Am J Respir Crit Care Med. 2005172(12):1529-33. [PubMed: 16020798]

13. Park SK, et al. HFE genotype, particulate air pollution, and heart rate variability: a geneenvironment interaction. Circulation. 2006; 114(25):2798-805. [PubMed: 17145987]

14. Dick CA, et al. Murine pulmonary inflammatory responses following instillation of sizefractionated ambient particulate matter. J Toxicol Environ Health A. 2003; 66(23):2193-2207. [PubMed: 14669776]

15. Rich DQ, et al. Are ambient ultrafine, accumulation mode, and fine particles associated with adverse cardiac responses in patients undergoing cardiac rehabilitation? Environ Health Perspect. 2012; 120(8):1162-9. [PubMed: 22542955]

16. Okin PM, et al. Repolarization abnormality for prediction of all-cause and cardiovascular mortality in American Indians: the Strong Heart Study. J Cardiovasc Electrophysiol. 2005; 16(9):945-51. [PubMed: 16174013]

17. Bauer A, et al. Heart rate turbulence: standards of measurement, physiological interpretation, and clinical use: International Society for Holter and Noninvasive Electrophysiology Consensus. J Am Coll Cardiol. 2008; 52(17):1353-65. [PubMed: 18940523]

18. Cygankiewicz I, et al. Heart rate turbulence predicts all-cause mortality and sudden death in congestive heart failure patients. Heart Rhythm. 2008; 5(8):1095-1102. [PubMed: 18675217]

19. Bigger JT Jr, et al. Correlations among time and frequency domain measures of heart period variability two weeks after acute myocardial infarction. Am J Cardiol. 1992; 69(9):891-8. [PubMed: 1550018]

20. Schneider A, et al. Changes in deceleration capacity of heart rate and heart rate variability induced by ambient air pollution in individuals with coronary artery disease. Part Fibre Toxicol. 2010; 7:29. [PubMed: 20929559]

21. Wang Y, et al. Long-term study of urban ultrafine particles and other pollutants. Atmospheric Environment. 2011; 45(40):7672-7680.

22. Tsuji $\mathrm{H}$, et al. Impact of reduced heart rate variability on risk for cardiac events. The Framingham Heart Study. Circulation. 1996; 94(11):2850-5. [PubMed: 8941112] 
23. Zuanetti G, et al. Prognostic significance of heart rate variability in post-myocardial infarction patients in the fibrinolytic era. The GISSI-2 results. Gruppo Italiano per lo Studio della Sopravvivenza nell' Infarto Miocardico. Circulation. 1996; 94(3):432-6. [PubMed: 8759085]

24. Pompella A, et al. The use of total antioxidant capacity as surrogate marker for food quality and its effect on health is to be discouraged. Nutrition. 2014; 30(7-8):791-3. [PubMed: 24984994]

25. Sies H. Total antioxidant capacity: appraisal of a concept. J Nutr. 2007; 137(6):1493-5. [PubMed: 17513413]

26. Hermans N, et al. Challenges and pitfalls in antioxidant research. Curr Med Chem. 2007; 14(4): 417-30. [PubMed: 17305543]

27. Niki E, Noguchi N. Evaluation of antioxidant capacity. What capacity is being measured by which method? IUBMB Life. 2000; 50(4-5):323-9. [PubMed: 11327327]

28. Baja ES, et al. Traffic-related air pollution and QT interval: modification by diabetes, obesity, and oxidative stress gene polymorphisms in the normative aging study. Environ Health Perspect. 2010; 118(6):840-6. [PubMed: 20194081]

29. Priori SG, et al. Evaluation of the spatial aspects of T-wave complexity in the long-QT syndrome. Circulation. 1997; 96(9):3006-12. [PubMed: 9386169]

30. Mordukhovich I, et al. Black carbon exposure, oxidative stress genes, and blood pressure in a repeated-measures study. Environ Health Perspect. 2009; 117(11):1767-72. [PubMed: 20049130]

31. Schneider A, et al. Endothelial dysfunction: associations with exposure to ambient fine particles in diabetic individuals. Environ Health Perspect. 2008; 116(12):1666-74. [PubMed: 19079718]

32. Zeger SL, et al. Exposure measurement error in time-series studies of air pollution: concepts and consequences. Environ Health Perspect. 2000; 108(5):419-26. [PubMed: 10811568] 
Highlight

- $\quad$ Ambient PM pollution associated with increased inflammation, blood pressure, \& HRV

- Total antioxidant capacity does not modify blood pressure responses to ambient PM

- $\quad$ Total antioxidant capacity does not modify inflammatory responses to ambient PM

- $\quad$ Total antioxidant capacity does not modify HRV responses to ambient PM

- Total antioxidant capacity does not modify T-wave complexity responses to ambient PM 


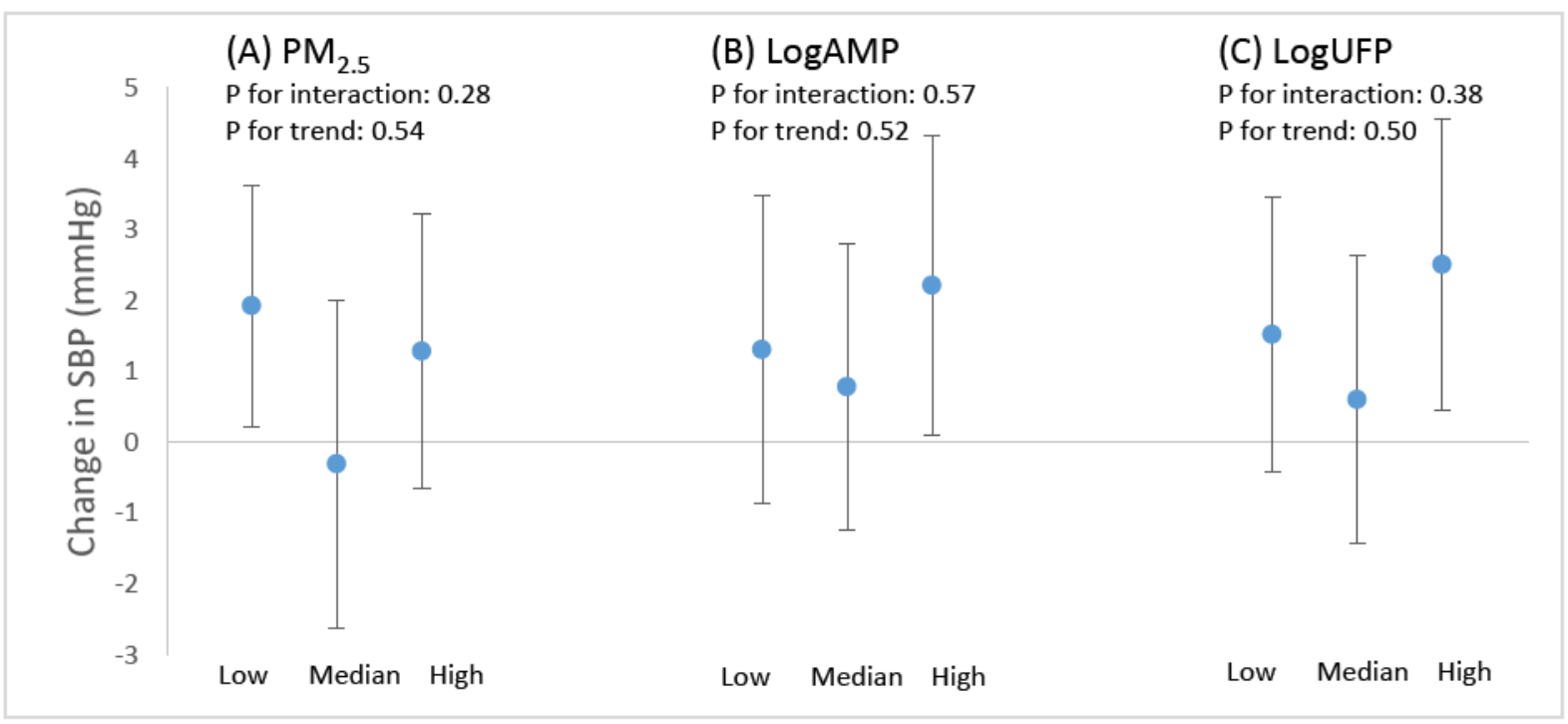

Figure 1.

Change in SBP ( $\mathrm{mmHg}$ ) measured at the beginning of the exercise session associated with each IQR increase in PM2.5 lagged 0-5 hours (A), log AMP lagged 0-23 hours (B), and log UFP lagged 24-47 hours (C), in the low, medium, and high TAC tertile group. 


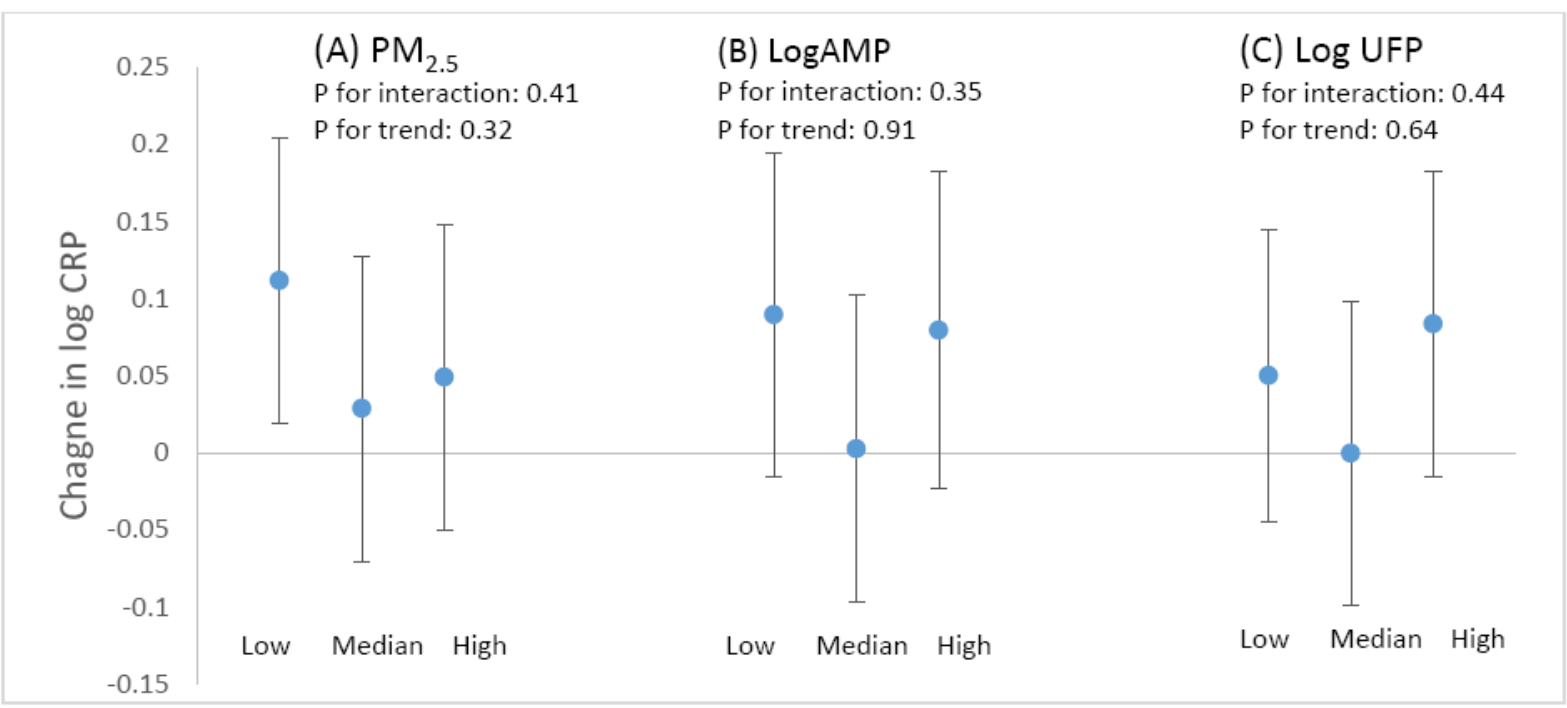

Figure 2.

Change in log CRP measured at the beginning of the exercise session associated with each IQR increase in PM2.5 lagged 0-23 hours (A), log AMP lagged 0-23 hours(B), and log UFP lagged 24-47 hours (C), in the low, medium, and high TAC tertile group. 


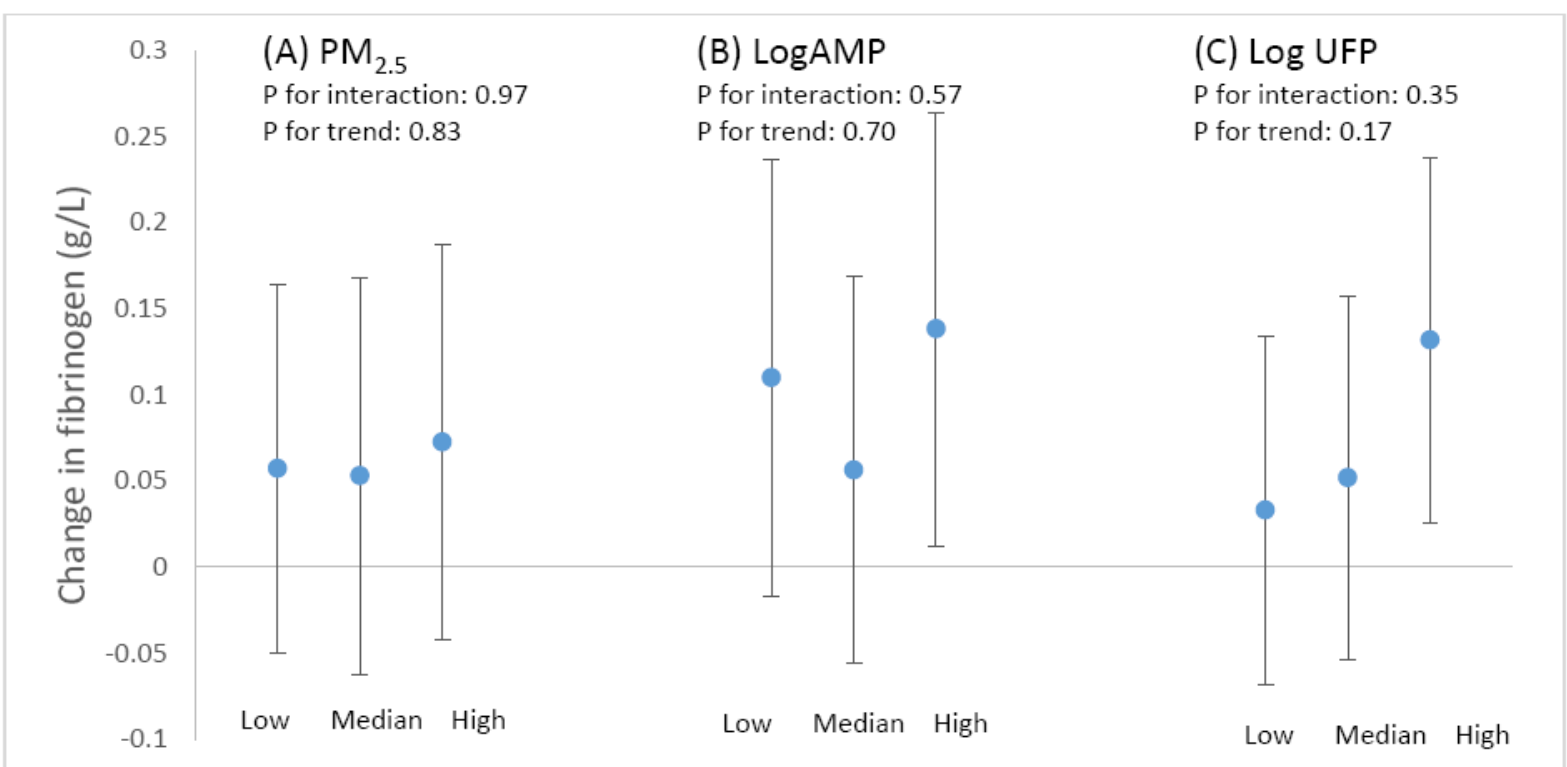

Figure 3.

Change in fibrinogen $(\mathrm{g} / \mathrm{L})$ measured at the beginning of the exercise session associated with each IQR increase in PM2.5 lagged 0-23 hours (A), log AMP lagged 24-47 hours(B), and log UFP lagged 24-47 hours (C), in the low, medium, and high TAC tertile group. 


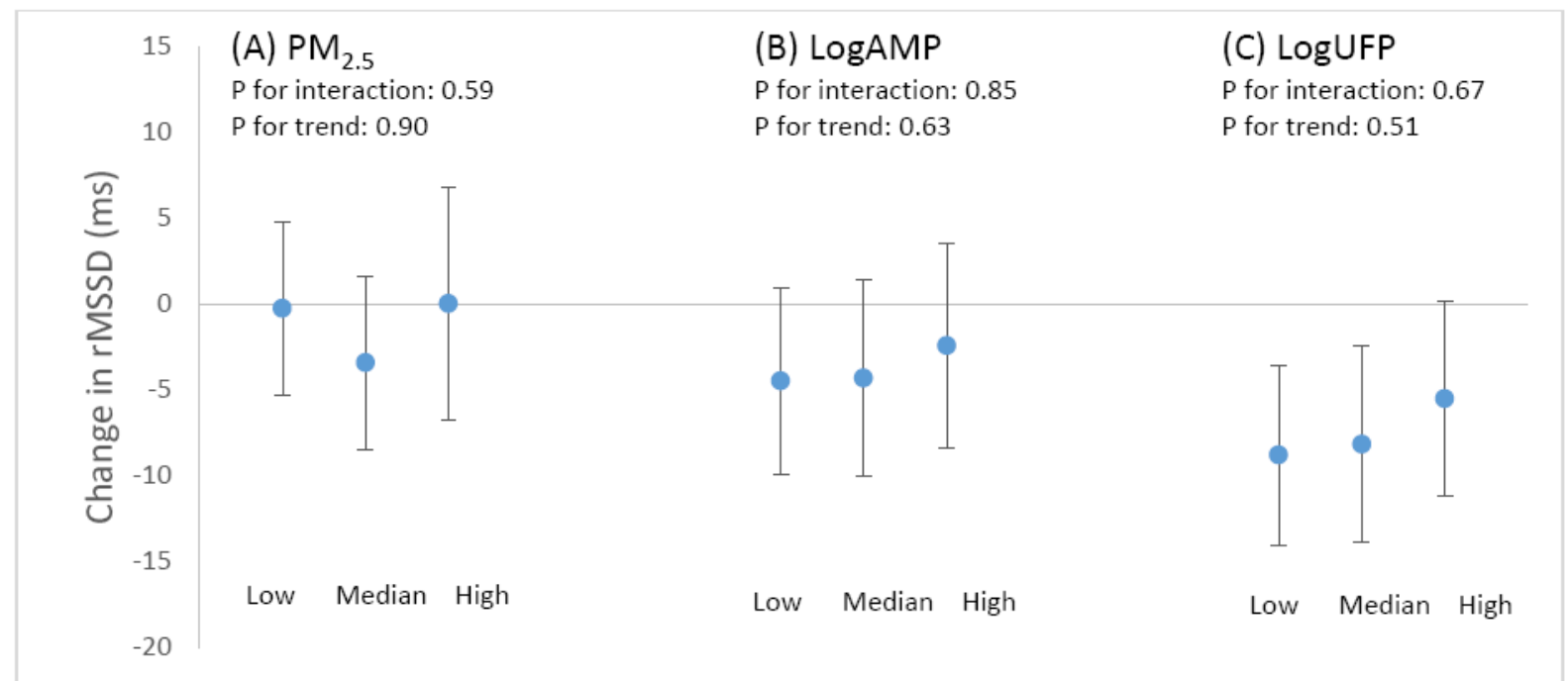

Figure 4.

Change in rMSSD (ms) measured across the whole session associated with each IQR increase in PM2.5 lagged 72-95 hours (A), log AMP lagged 72-95 hours (B), and log UFP lagged 72-95 hours (C), in the low, medium, and high TAC tertile group. 


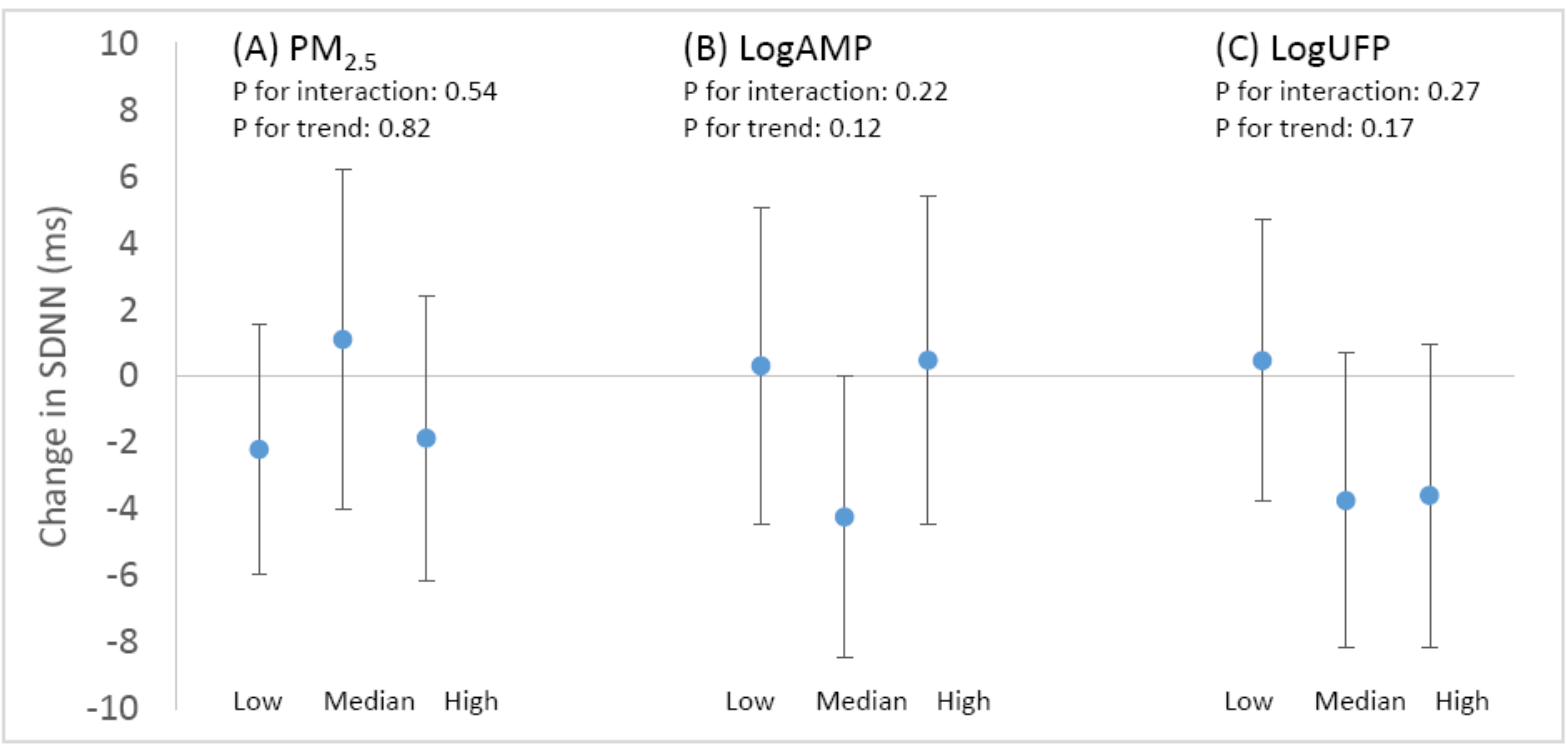

Figure 5.

Change in SDNN (ms) measured across the whole session associated with each IQR increase in PM2.5 lagged 0-5 hours(A), log AMP lagged 0-5 hours (B), and log UFP lagged 24-47 hours (C), in the low, medium, and high TAC tertile group. 


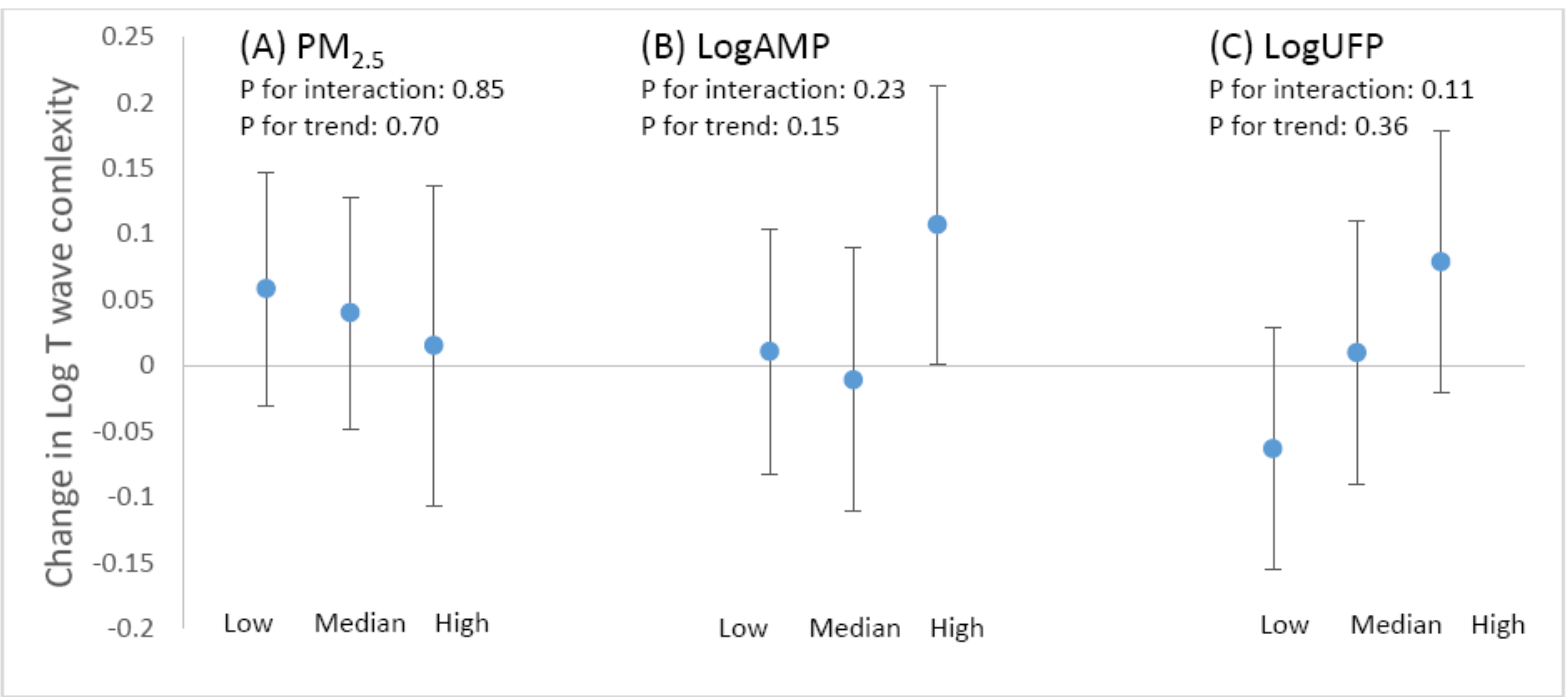

Figure 6.

Change in $\log \mathrm{T}$ wave complexity measured in the pre-exercise resting period associated with each IQR increase in PM2.5 lagged 72-95 hours (A), log AMP lagged 72-95 hours(B), and log UFP lagged 72-95 hours (C), in the low, medium, and high TAC tertile group. 
Table 2

Change in each outcome associated with each IQR increase in mean $\mathrm{PM}_{2.5}, \log$ AMP, and log UFP concentrations, in the previous 120 hours.

\begin{tabular}{|c|c|c|c|}
\hline & $\mathrm{PM}_{2.5}{ }^{a}$ & $\log \mathrm{AMP} b$ & $\log \mathrm{UFP}^{c}$ \\
\hline & Change in outcome (95\% CI) & Change in outcome (95\% CI) & Change in outcome $(95 \% \mathrm{CI})$ \\
\hline \multicolumn{4}{|c|}{ SBP (mmHg) } \\
\hline $0-5$ & $1.27(0.09,2.46)^{*}$ & $0.82(-0.48,2.12)$ & $-0.31(-1.51,0.88)$ \\
\hline $0-23$ & $0.69(-0.67,2.06)$ & $1.48(0.09,2.86)^{*}$ & $1.38(0.07,2.68)^{*}$ \\
\hline $24-47$ & $-0.36(-1.62,0.90)$ & $0.61(-0.89,2.11)$ & $1.60(0.32,2.89)^{*}$ \\
\hline $48-71$ & $0.49(-0.86,1.84)$ & $1.06(-0.36,2.47)$ & $0.97(-0.38,2.32)$ \\
\hline $72-95$ & $0.09(-1.15,1.33)$ & $0.70(-0.60,2.01)$ & $0.52(-0.74,1.78)$ \\
\hline $96-119$ & $-0.20(-1.28,0.88)$ & $0.07(-1.27,1.41)$ & $-0.30(-1.65,1.05)$ \\
\hline \multicolumn{4}{|c|}{$\log$ CRP } \\
\hline $0-5$ & $0.047(-0.006,0.100)^{\#}$ & $0.050(-0.013,0.113)$ & $0.033(-0.024,0.091)$ \\
\hline $0-23$ & $0.065(0.003,0.126)^{*}$ & $0.051(-0.017,0.119)$ & $0.039(-0.024,0.102)$ \\
\hline $24-47$ & $0.010(-0.047,0.068)$ & $0.040(-0.034,0.114)$ & $0.041(-0.021,0.104)$ \\
\hline $48-71$ & $0.015(-0.048,0.077)$ & $-0.035(-0.104,0.034)$ & $-0.033(-0.098,0.032)$ \\
\hline $72-95$ & $0.051(-0.007,0.108)^{\#}$ & $0.001(-0.063,0.064)$ & $-0.022(-0.084,0.039)$ \\
\hline $96-119$ & $0.016(-0.034,0.065)$ & $0.000(-0.065,0.066)$ & $0.008(-0.057,0.074)$ \\
\hline \multicolumn{4}{|c|}{ Fibrinogen $(g / L)$} \\
\hline $0-5$ & $0.02(-0.04,0.08)$ & $0.01(-0.06,0.08)$ & $0.02(-0.04,0.08)$ \\
\hline $0-23$ & $0.06(-0.01,0.13)$ & $0.06(-0.02,0.13)$ & $0.04(-0.03,0.11)$ \\
\hline $24-47$ & $0.05(-0.02,0.11)$ & $0.10(0.02,0.18)^{*}$ & $0.07(0.00,0.14)^{*}$ \\
\hline $48-71$ & $0.06(-0.01,0.13)$ & $0.06(-0.02,0.13)$ & $0.05(-0.03,0.11)$ \\
\hline 72-95 & $0.01(-0.06,0.07)$ & $0.05(-0.02,0.12)$ & $0.03(-0.03,0.10)$ \\
\hline $96-119$ & $-0.04(-0.10,0.01)$ & $0.01(-0.06,0.08)$ & $0.04(-0.03,0.11)$ \\
\hline \multicolumn{4}{|c|}{ rMSSD (ms) } \\
\hline $0-5$ & $1.19(-1.89,4.28)$ & $-1.67(-5.14,1.79)$ & $-3.58(-6.77,-0.39)^{*}$ \\
\hline $0-23$ & $1.57(-1.97,5.12)$ & $-1.95(-5.64,1.74)$ & $-3.71(-7.18,-0.25)^{*}$ \\
\hline $24-47$ & $1.41(-1.86,4.68)$ & $-0.90(-4.90,3.09)$ & $-4.40(-7.81,-0.99)^{*}$ \\
\hline $48-71$ & $0.16(-3.34,3.66)$ & $-1.82(-5.60,1.96)$ & $-4.36(-7.90,-0.82)^{*}$ \\
\hline 72-95 & $-1.27(-4.54,1.99)$ & $-3.54(-7.02,-0.06)^{*}$ & $-7.48(-10.77,-4.20)^{* *}$ \\
\hline $96-119$ & $-0.18(-3.00,2.63)$ & $-1.46(-5.04,2.12)$ & $-2.31(-5.91,1.28)$ \\
\hline \multicolumn{4}{|c|}{ SDNN (ms) } \\
\hline $0-5$ & $-1.32(-3.74,1.11)$ & $-1.51(-4.37,1.34)$ & $-0.95(-3.57,1.68)$ \\
\hline $0-23$ & $-0.82(-3.85,2.21)$ & $-1.05(-4.10,2.01)$ & $-1.14(-4.00,1.71)$ \\
\hline $24-47$ & $-0.14(-2.95,2.66)$ & $-0.69(-4.00,2.61)$ & $-2.14(-4.95,0.67)$ \\
\hline $48-71$ & $1.97(-1.04,4.97)$ & $1.50(-1.63,4.63)$ & $-0.24(-3.17,2.70)$ \\
\hline 72-95 & $0.33(-2.44,3.10)$ & $-1.44(-4.33,1.45)$ & $-0.71(-3.46,2.04)$ \\
\hline
\end{tabular}




\begin{tabular}{lccc}
\hline & \multicolumn{1}{c}{$\mathbf{P M}_{\mathbf{2 . 5}}{ }^{\boldsymbol{a}}$} & $\operatorname{logAMP}^{b}$ & $\operatorname{logUFP}^{\boldsymbol{c}}$ \\
\cline { 2 - 4 } & Change in outcome (95\% CI) & Change in outcome (95\% CI) & Change in outcome (95\% CI) \\
\hline $96-119$ & $0.69(-1.70,3.08)$ & $-1.33(-4.31,1.64)$ & $-0.81(-3.78,2.17)$ \\
Log T wave complexity & & & \\
$0-5$ & $-0.010(-0.065,0.046)$ & $-0.034(-0.094,0.026)$ & $-0.036(-0.090,0.019)$ \\
$0-23$ & $-0.018(-0.082,0.046)$ & $-0.059(-0.123,0.005)$ & $-0.042(-0.102,0.017)$ \\
$24-47$ & $0.006(-0.053,0.065)$ & $-0.023(-0.092,0.047)$ & $-0.046(-0.105,0.014)$ \\
$48-71$ & $0.025(-0.038,0.088)$ & $0.017(-0.049,0.083)$ & $-0.001(-0.063,0.061)$ \\
$72-95$ & $0.046(-0.011,0.104)$ & $0.029(-0.032,0.089)$ & $0.004(-0.054,0.062)$ \\
$96-119$ & $0.002(-0.048,0.052)$ & $0.016(-0.046,0.078)$ & $-0.009(-0.071,0.053)$ \\
\hline
\end{tabular}

${ }^{a} \mathrm{IQR}$ increases of $7.2 \mathrm{ug} / \mathrm{m}^{3}$ (6-hour mean) and $6.7 \mathrm{ug} / \mathrm{m}^{3}$ (24-hour) mean.

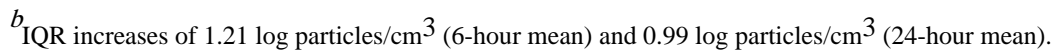

${ }^{c}$ IQR increases of $0.87 \mathrm{log}$ particles $/ \mathrm{cm}^{3}$ (6-hour mean) and $0.81 \mathrm{log}$ particles $/ \mathrm{cm}^{3}$ (24-hour) mean.

${ }_{\mathrm{P}<0.1}$

* $\mathrm{P}<0.05$,

*** $<<0.01$. 
